to the organic matter in the soil. It is unfortunate that no full description of any of the sites or soils examined is published although it may have been recorded elsewhere. It has been found that Sitka spruce is especielly susceptible to attack, and not solely because it happens to occur in no fewer than 38 of the 58 areas examined. A point which is not made clear is whether Rhizina exists in the soil before the plantation is established-possibly associated with the organic matter therein-and Sitka spruce is most often planted on soils with a high content of organic matter or on grass- or rush-covered sites. Rhizina may exist in close association with grasses, for example, and only when the organic matter is altered or consumed by fire does it virulently attack the feeding roots of the trees. What, for example, happens when Rhizina encounters the mycorrhizal fungi usually associated with conifers? There is a great need for more research into the occurrence and behaviour of all the micro-organisms found in the soil, and it is important for foresters that soil research should be based, where possible, on the soil of the natural forest. While the natural forest is a reality which has stood the test of time, the artificial forest still remains an illusory concept nowhere brought to satisfactory permanency, and liabls to be seriously disturbed by organisms. like Rhizina, 'beyond the forester's control.

\title{
TRENDS IN JUVENILE DELINQUENCY
}

$\mathrm{F}^{\circ}$ OR some years the World Health Organization has taken an interest in the problem of juvenile delinquency. In 1949, at the request of the United Nations, the Organization carried out a study of the psychiatric aspects of the origin, prevention and treatment of juvenile delinquency as a contribution to the United Nations programme for the prevention of crime and treatment of offenders. Since then, representatives of the World Health Organization have taken part in many conferences and have regularly attended meetings of the United Nations Consultative Group on the Prevention of Crime and the Treatment of Offenders; and the Organization has, through its Regional Office for Europe in Copenhagen, organized a seminar on the psychiatric treatment of offenders.

In 1959, again at the request of the United Nations, another study was carried out by a psychiatric consultant, Dr. T. C. N. Gibbens, senior lecturer in forensic psychiatry in the University of London, who was appointed by the World Health Organization to complement the earlier study with a review of present trends in juvenile delinquency, based on his own personal experience, the recent literature and first-hand information obtained during visits to a number of countries-Australia, Denmark, the Federal Republic of Germany, Israel, Lebanon, Poland, Sweden and Yugoslavia. Dr. Gibbens's report, which was submitted to the second United Nations Congress on the Prevention of Crime and Treatment of Offenders, has now been published with some minor amendments *.

The new forms or manifestations of juvenile delinquency consist partly of delinquent acts which are a consequence of new opportunities for crime, but which do not differ in their essential character from more traditional forms, and partly of offences which appear to reveal a fundamental change in behaviour, or the participation of sections of the community which have not previously been involved in crime.

It is with these newer forms that the author is primarily concerned in his review of social changes, changes in family life and in the individual delinquent, special property offences, sexual offences, wayward girls, violent offences, alcoholism and drug addiction, and hooliganism. He also discusses present trends in prevention, paying particular attention to the development of prediction studies designed to identify young children who are in danger of being seriously delinquent in the future, and to the tendency to design preventive programmes in such a way that their results can be scientifically evaluated. A chapter on treatment deals with diagnosis, treatment at liberty and institutional treatment.

* World Health Organization Public Health Papers. No. 5: Trends in Juvenile Delinquency. By T. C. N. Gibbens. Pp. 56. 2 Swiss francs; $38.6 d . ; 0.60$ dollar. (Geneva: World Health Organization; London: H.M.S.O., 1961).

\section{SIMIAN MALARIA}

\begin{abstract}
A RECENT issue of the World Health Organization Chronicle is concerned with the transmission of malaria parasites between monkey and man*.

The knowledge that such a transmission can take place dates back to 1932 , when, in India, Knowles and Das Gupta found that Plasmodium knowlesi could cause a febrile infection in man. Knowledge of simian malaria in relation to man was extended in 1948 , when $P$. rodheini of African chimpanzees was found to be morphologically and immunologically identical with $P$. malariae of man. Subsequent work showed that human malaria parasites such as $P$. vivax, $P$. falciparum and $P$. ovale could be transmitted to chimpanzees through anopheline mosquitoes although

* World Health Organization Chronicle, 15, No. 1, January 1961.
\end{abstract}

patent parasitæmia appeared only in splenectomized apes.

In certain parts of West Africa the chimpanzee is known to harbour $P$. malariae, and man has been shown to be readily susceptible to the strains har. boured. The anopheline vector of the $P$. malariae of these chimpanzees has not been identified. In Sarawak certain strains of human parasites of quartan malaria have been observed to have a peculiar morphology which suggested that the parasite might be of simian origin. In the Amazon region of South America, some $P$. vivax infections in man have been held to be derived from simian malaria. $P$. simium, found in howler monkeys of the forests near Santos, has been suspected of being closely related to $P$. ovale. 
In May 1960, came a new development. During research at the U.S. National Institutes of Health, Bethesda, by Eyles, Coatney and Getz on the transmission to rhesus monkeys of $P$. cynomolgi bastianelli, some very large-scale inoculations of sporozoites into monkeys were carried out. Two staff members of the Institutes, neither of whom had had any recent contact with human malaria, were dealing at close quarters with heavily infected mosquitoes; they both developed illness with fever which, after a remittent period, proved to have tertian periodicity. Blood smears, made on new slides and stained with new stain in clean dishes, showed that both had vivax-type parasites with enlarged erythrocytes and Schuffner's stippling. Five millilitres of blood from each of the two individuals, injected intravenously into two uninfected rhesus monkeys, produced cynomolgi-type infections in 8 days in one monkey and in 10 days in the other. Two human volunteers each received $10 \mathrm{ml}$. of blood intravenously from one of the individuals; both developed clinical attacks of malaria with vivaxtype parasites, enlarged erythrocytes, and Schuffner's stippling, but without high parasite densities.

The two individuals in whom the infection was first discovered received chloroquine treatment within 24 hr. of diagnosis. At the time of treatment both showed low levels of parasitæmia. The parasites promptly disappeared with treatment and no relapse occurred.

Another person allowed 30-50 Anopheles freebornii, heavily infected with $P$. cynomolgi bastianelli, to feed on him, and experienced headache, malaise and fever eleven and twelve days later. No parasites were found in his blood, but a monkey free of disease, intravenously injected with $10 \mathrm{ml}$. of his blood, developed an overt infection in 6 days. A fourth person developed a typical malaria paroxysm with vivax-type parasites fourteen days after being bitten by $10 A$. freebornii, heavily infected with $P$. cynomolgi bastianelli.

Cross-immunity studies were undertaken at Bethesda on two monkeys which had recovered spontaneously from infections with $P$. cynomolgi bastianelli. On being re-inoculated with hesvy doses of parasites taken from one of the two monkeys which had been infected by intravenous injection of the blood of one of the two accidentally infected staff members, one monkey showed a transient parasitæmia indicating almost complote immunity. The other had an attack of malaria that was shorter and less severe than the usual bastianelli attack. These results supported the view that the human infections were due to $P$. cynomolgi bastianelli.

The significance of these infections of human beings lies in the fact that they were transmitted by mosquitoes which had fed on rhesus monkeys. The theoretical possibility of natural infection of man with monkey malaria had already been suspected; but it was the anthropoid ape, not the monkey, which was formerly suspected as being a possible carrier of human malaria. Monkeys are much more numerous and widespread than apes, and live in eloser association with man, and at lesst seven species of anophelines are known to be able to transmit $P$. cynomolgi to rhesus monkeys. Some of these species are exophilic, and therefore unlikely to be affected by residual insecticides; moreover, the host preferences of anopheline vectors for man and for the monkey are often almost the same. Consequently, experimental proof that man can be infected by monkey malaria opens up a new range of possibilities.

\title{
EFFECT OF MASS TRANSFER ON THE VELOCITY OF RISE OF BUBBLES IN WATER
}

\author{
By Dr. J. H. LEONARD and Prof. G. HOUGHTON \\ Department of Chemical Engineering, University of Pittsburgh, Pennsylvania
}

\begin{abstract}
A LTHOUGH Datta, Napier and Newitt ${ }^{1}$ were the first to suggest that "mass transfer (from bubbles) may be accompanied by some retarding effect", no definite conclusions were reached in this respect from their experiments on carbon dioxide bubbles rising in water. In the present measurements both velocities of rise and mass transfer-rates for bubbles could be measured separately over short distances in a column of water. The apparatus used was a modification of that ${ }^{2}$ in which soluble bubbles were formed in a cup under mercury and allowed to rise up through a completely closed 'Pyrex' glass column $(9 \mathrm{~cm}$. internal diameter, $270 \mathrm{~cm}$. long) filled with water; the change in volume of each bubble was recorded by registering the position of a mercury meniscus in a horizontal capillary side-arm, which provided the only opening to the atmosphere. By stretching a fine platinum wire through the capillary and connecting the wire to a bridge circuit it was possible to record the position of the mercury meniscus and hence the bubble volume on one channel of a high-speed Sanborn oscillograph. Although the principle was used earlier ${ }^{3,4}$, these earlier experimenters used high-speed photography to determine
\end{abstract}

the volume of the bubble by photographing the position of the liquid meniscus in the capillary. However, the present apparatus also differ's from that of Houghton et al. ${ }^{2}$ in that the rising bubbles interrupt a series of light beams and photoelectric cells placed at intervals of $47.0 \mathrm{~cm}$. up the column. As the bubble passed each light beam, the change in light intensity was recorded on a second channel of the Sanborn oscillograph. In this way it was possible to determine the average bubble velocity and the average mass transfer-rate separately over each $47 \cdot 0-\mathrm{cm}$. section of the column together with the corresponding average bubble diameter over the same section. In certain instances the velocities of rise, obtained by the method described, were also confirmed independently in a separate apparatus by forming the bubbles at jets of various sizes, using a micrometer hypodermic syringe, and then timing them over a $110 . \mathrm{cm}$. length of column, using a stopwatch and starting the measurements at a distance of $20 \mathrm{~cm}$. from the jet (af. ref. 5).

Figs. 1 and 2 show that mass transfer has a profound effect on the velocity of rise of soluble gases in water for bubbles with equivalent spherical diameters 